\title{
Grb2-associated binder 2 expression and its roles in uveal melanoma invasion
}

\author{
MEILING CHEN ${ }^{1 *}$, YUEHUA LI $^{2 *}$, XIUNING SUN ${ }^{3}$, BAOGANG ZHANG $^{4}$, WEI LI $^{5}$, \\ SHUXIAO WANG ${ }^{1}$, XUETAO ZHU $^{1},{\text { FENG } \mathrm{LI}^{1} \text { and LIHONG SHI }}^{1}$ \\ ${ }^{1}$ Department of Pharmacology, Weifang Medical University, Weifang 261053; ${ }^{2}$ Department of Ophthalmology, \\ Beijing Chaoyang Hospital, Capital Medical University, Beijing 100020; Departments of ${ }^{3}$ Microbiology and ${ }^{4}$ Pathology, \\ Weifang Medical University, Weifang 261053; ${ }^{5}$ Department of Gynecology, Zhenjiang Maternity and Child Health Hospital, \\ Zhenjiang 212001, P.R. China
}

Received March 13, 2017; Accepted July 25, 2017

DOI: $10.3892 / \mathrm{mmr} .2017 .7151$

\begin{abstract}
Uveal melanoma (UM) is characterized by high metastasis and poor prognosis. A more improved understanding of the metastatic mechanism in UM cells is essential for the design of molecular therapy. Grb2-associated binder 2 (Gab2) has been reported to serve important roles in the progression of various types of human cancer. However, the role of Gab2 in the migration and invasion of UM remains unclear. The present study sought to further assess the expression of Gab2 in UM and the role of Gab2 in the invasion of UM cells. Clinical UM tissue samples and UM cell lines were analyzed using western blot analysis for the expression of Gab2. RNA interference was used to investigate the effect of Gab2 on the migratory and invasive characteristics of UM cells in vitro. The expression levels of matrix metalloproteinase (MMP)2, MMP9 and fascin in Gab2-knockdown, and control cells were also detected using western blot analysis. A total of 20 clinical UM samples and a subset of UM cell lines were investigated with uniformly high Gab2 expression. In the in vitro experiment, reduction of Gab2 using small interfering RNA inhibited the migration and invasion of UM cells by mediating MMPs, and fascin expression. These data suggest that Gab2 is a useful prognostic marker for UM and a novel therapeutic target for UM metastasis intervention.
\end{abstract}

Correspondence to: Professor Lihong Shi, Department of Pharmacology, Weifang Medical University, 7166 Baotongxi Street, Weifang 261053, P.R. China

E-mail: shilih@163.com

*Contributed equally

Abbreviation: Gab2, Grb2-associated binder 2

Key words: Gab2, uveal melanoma, invasion

\section{Introduction}

Uveal melanoma (UM) is the most common primary intra-ocular malignancy in adults that accoubts for $70 \%$ of all eye malignancies. Up to $50 \%$ of UM patients are at risk of metastasis (1), and the median survival time from the identification of metastasis varies from <4-12.5 months (2). Despite the improvements in diagnosis and treatment of the primary tumor, the prognosis of UM patients remains poor (3). Basing on potential biological targets from known pathways implicated in tumor progression, the researchers found that targeted molecular therapies are possible in the treatment of UM and may result in improved patient survival (4).

Previous studies have suggested that Gab2 is involved in tumor migration and invasion. As a member of the DOS/Gab family of scaffolding adapters, Gab2 possesses a C-terminal portion with numerous tyrosine phosphorylation sites and a N-terminal pleckstrin homology domain and proline-rich motifs (5). On stimulation, Gab2 interacts with SH2-domain-containing proteins and mediates signals that can promote migration $(6,7)$. Gab2 can regulate the migratory behaviors by activating the PI3K pathway in ovarian cancer and glioma $(8,9)$. In addition, a high expression of Gab2 was detected in gastric (10), lung (11), and breast cancer. Moreover, Gab2-deficient cancer cells exhibited decreased migration ability, which strongly suggested that upregulated Gab2 is associated with tumor metastasis (9). However, the role of Gab2 in UM remains unclear. In this study, we investigated the expression of Gab2 in UM and its role in the invasion of $\mathrm{UM}$ cells in vitro.

\section{Materials and methods}

Patients and tissue specimens. Twenty pairs of randomly selected frozen (liquid nitrogen) UM tissues were taken from 20 patients who underwent surgical treatment for primary UM with pathological identification in Beijing Chaoyang Hospital (Beijing, China) between 2011 and 2015, guided by a protocol approved by the Institutional Review Board. Patients gave consent for the use of their tissue specimens in the present study. None of them received chemotherapy or radiotherapy 
before surgery. A total of 13 males and 7 females were included in the study, ranging in age from 34 to 67 years (median age 51 years).

Cell culture and reagents. The human normal retinal pigment epithelium cell line ARPE19 and three UM cell lines MEL 202, 92.1, and MEL 270 were obtained from the American Type Culture Collection (Manassas, VA, USA). ARPE19 cells were maintained in F-12 supplemented with $10 \%$ fetal bovine serum (FBS). The UM cell lines MEL202, 92.1, and MEL270 were grown in RPMI medium (HyClone; GE Healthcare Life Sciences, Logan, UT, USA) supplemented with 10\% FBS (SH30070.03; HyClone), penicillin (100 $\mu \mathrm{g} / \mathrm{ml}$; Invitrogen, Carlsbad, CA, USA), and streptomycin (100 $\mu \mathrm{g} / \mathrm{ml}$; Invitrogen). MEL270 and MEL202 were additionally supplemented with $1 \%$ minimal essential medium (MEM) vitamin solution and 1\% MEM non-essential amino acids (Invitrogen). Cells were incubated at $37^{\circ} \mathrm{C}$ in a humidified atmosphere containing $5 \% \mathrm{CO}_{2}$ and split when they reached $90-95 \%$ confluence. The chemotaxis chambers and membranes were from Neuroprobe (Gaithersburg, MD). The human epidermal growth factor (EGF) was purchased from R\&D systems (Minneapolis, MN, USA). Bovine serum albumin (BSA) was purchased from Amresco (0332; Amresco, Solon, OH, USA).

Plasmid construction, small interfering RNA, and plasmid transfection. Cells were plated in a $35-\mathrm{mm}$ dish for $24 \mathrm{~h}$ before transfection into the complete medium. Transfection was performed with Lipofectamine 2000 (Invitrogen), according to the manufacturer's instructions. Stealth small interfering RNA (siRNA) against human Gab2 (5'-GTGAGAACGATG AGAAATA-3') and a scrambled siRNA were synthesized by Invitrogen. BLOCK-iT Fluorescent Oligo was used to examine the transfection efficiency (Invitrogen). Cells were harvested after $72 \mathrm{~h}$ and used for further experiments. Stable transfectants were selected by using $600 \mu \mathrm{g} / \mathrm{ml}$ neomycin.

Chemotaxis assay. Chemotaxis assays were performed by using Transwell chambers as described previously (12). Briefly, the chemo-attractant (EGF) was loaded into the lower chemotaxis chamber and $5 \times 10^{5}$ cells $/ \mathrm{ml}$ cells suspended in the binding medium were added to the upper chambers. The polycarbonate filter $(8-\mu \mathrm{m}$ pore size) was pretreated with $10 \mu \mathrm{g} / \mathrm{ml}$ fibronectin overnight, dried in air, and inserted between the upper and lower chambers. Then, the chamber was incubated at $37^{\circ} \mathrm{C}$ in $5 \% \mathrm{CO}_{2}$ for $3 \mathrm{~h}$. The filter membrane was then rinsed, fixed, and stained. The numbers of migrating cells were counted at $\mathrm{x} 400$ in three separate fields by light microscope.

Scratch assay. The cells were plated in 35-mm dishes for two days to grow into a monolayer. Then, the dishes were outlined with an even trace in the middle using a $10-\mu 1$ pipette tip. The cells were then incubated at $37^{\circ} \mathrm{C}$ in $5 \% \mathrm{CO}_{2}$ within an appropriate time, and the distance of the wounds was measured under a light microscope. All samples were tested in triplicate, and the data are expressed as the mean \pm SD.

Matrigel invasion assay. The invasion of cells in vitro was measured through Matrigel-coated Transwell inserts (Costar,
Cambridge, MA) (13). Briefly, the Transwell inserts with 8- $\mu \mathrm{m}$ pore sizes were coated with a final concentration of $1.5 \mathrm{mg} / \mathrm{ml}$ of Matrigel. The cells were trypsinized and $200 \mu \mathrm{l}$ of cell suspension $\left(5 \times 10^{5}\right.$ cells $\left./ \mathrm{ml}\right)$ were added in triplicate wells. A total of $300 \mu \mathrm{l}$ of binding medium with $10 \mathrm{ng} / \mathrm{ml}$ of EGF was added to the lower well. After $24 \mathrm{~h}$ of incubation $\left(37^{\circ} \mathrm{C}\right.$, $5 \% \mathrm{CO}_{2}$ ), the non-invading cells were removed by wiping the upper side of the membrane, and the invading cells were fixed and stained. The number of invading cells was counted under a microscope (IX71; Olympus Corporation, Tokyo, Japan) in five predetermined fields (CellSens Standard; total magnification, x400). All assays were independently repeated at least three times. The differences in the invasion rates between control and Gab2-deficient cells were analyzed using two-tailed Student's test.

Enzyme-linked immunosorbent assay. Enzyme-linked immunosorbent assay (ELISA) was performed to determine the MMP-2 and MMP-9 expression levels in the culture medium of control and Gab2 knockdown cells. Cells $\left(2 \times 10^{5}\right.$ cells/well each) were plated onto six-well plates in RPMI-1640 containing 5\% FBS. The cells were allowed to grow for $48 \mathrm{~h}$ until they were approximately $60-70 \%$ confluent. The growth medium was then removed and replaced with fresh RPMI-1640 containing 1\% FBS. A concentration of $10 \mathrm{ng} / \mathrm{ml}$ EGF was added to the growth medium to stimulate the expression of MMP-2 and MMP-9. The cells were further incubated for $24 \mathrm{~h}$ until approximately $80 \%$ confluence was attained. The medium was then harvested and filtered for the measurement of MMP-2 and MMP-9 using an ELISA kit (R\&D Systems, USA) according to the manufacturer's instructions. The remaining cells were directly lysed in $1 \mathrm{x}$ sodium dodecyl sulfate (SDS) sample buffer and protein samples were for western blot assay. The optical density of each well was measured using an automated microplate reader $\left(E_{\text {Max }}\right.$; Molecular Devices, LLC, Sunnyvale, CA, USA). Each experiment was performed three times, and the mean concentrations of MMP-2 and MMP-9 were presented as the final results.

Western blot analysis. For western blot analysis, protein samples were separated by SDS-polyacrylamide gel electrophoresis, transferred to polyvinylidene difluoride membranes (Millipore Inc., Billerica, MA, USA), immunoblotted using the appropriate primary and the horse radish peroxidase (HRP) conjugated secondary antibodies, and were visualized by using enhanced chemiluminescence reagents ECL (Pierce, Rockford, IL, USA). Western blot data shown in the paper are representatives from three independent experiments. The intensities of the bands in western blots were quantified by densitometry analysis using AlphaImager HP (ProteinSimple, San Jose, CA, USA) and NIH Image J software (Rockville, MD, USA). The following commercial antibodies were used in the present study: Gab2 (sc-25498, 1:1,000 dilution; Santa Cruz Biotechnology, Inc., Santa Cruz, CA, USA), MMP-2 (4022, 1:1,000 dilution; Cell Signaling, Technology, Inc., Danvers, MA, USA), MMP-9 (3852, 1:1,000 dilution; Cell Signaling Technology, Inc.), fascin (Santa Cruz, sc28265, 1:1,000 dilution), $\beta$-actin (4970, 1:1,000 dilution; Cell Signaling Technology, Inc.), and HRP-linked Anti-rabbit IgG 
$\mathbf{A}$

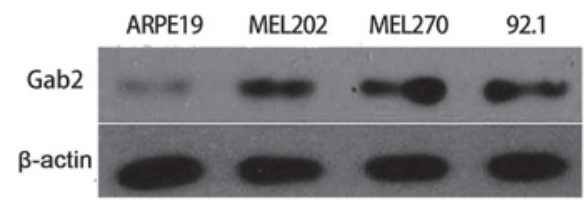

D

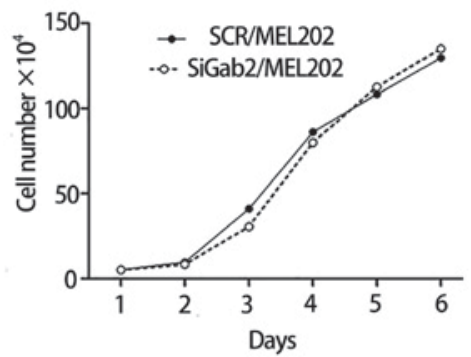

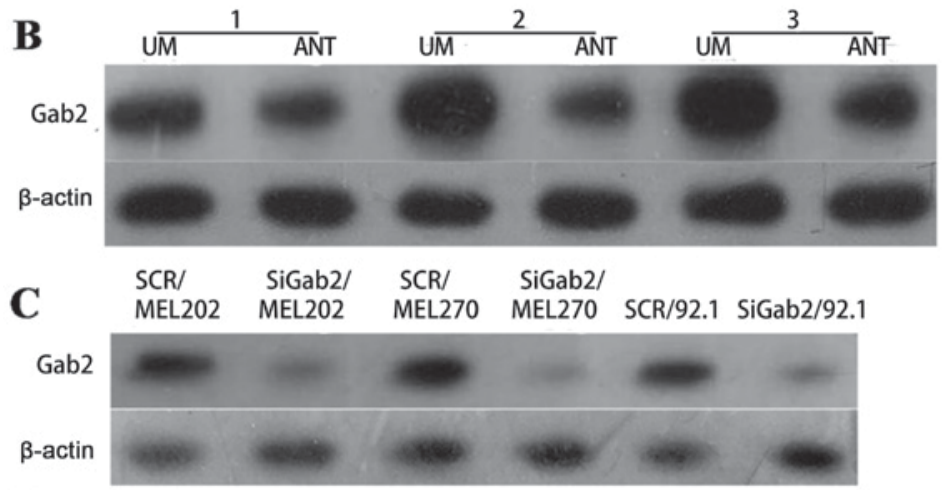

E

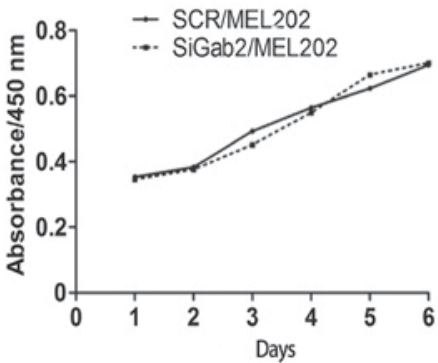

Figure 1. Analysis of Gab2 expression in UM cell lines and primary UM tissues. (A) Western blot analysis of Gab2 protein in UM cell lines (MEL202,92.1 and MEL270) and the human normal retinal pigment epithelium cell line ARPE19. (B) Western blot analysis of Gab2 protein expression in primary UM tissues (T) and their paired ANT. (C) Western blot analysis of Gab2 protein expression in SCR/MEL202, siGab2/MEL202, SCR/92.1, siGab2/92.1, SCR/MEL270, and siGab2/MEL270 cells. $\beta$-actin was used as a loading control. (D and E) Cell proliferation and XTT assay of SCR/MEL202 and siGab2/MEL202 cells, respectively. Each data point was an average of triplicate assays.

antibody (7074, 1:2,000 dilution; Cell Signaling Technology, Inc.).

Statistical analysis. All statistical analyses were performed using the SPSS 18.0 statistical software package (SPSS, Inc. Chicago, IL, USA). In all cases, $\mathrm{P}<0.05$ was considered to indicate a statistically significant difference.

\section{Results}

Upregulation of Gab2 in UM cell lines and primary UM tissues. Western blot analyses on primary UM cell lines and tissues showed that the protein levels of Gab2 were higher in three UM cell lines 92.1, MEL270, and MEL202 compared with human normal retinal pigment epithelium cell line ARPE19 (Fig. 1A). Similarly, the protein levels of Gab2 were higher in tumor tissues (T) than that in adjacent non-tumor tissues (ANT) (Fig. 1B). These results clearly showed notable upregulation of Gab2 in UM. We obtained stable Gab2 knockdown UM cells siGab2/MEL202, siGab2/92.1, siGab2/MEL270 and the RNAi control cells SCR/MEL202, SCR/92.1 and SCR/MEL270 (Fig. 1C). We detected the proliferation rates of these above-mentioned cells in vitro. The proliferation assay showed that the knockdown of Gab2 did not significantly influence cell proliferation (Fig. 1D), and they have been verified by XTT expression (Fig. 1E).

Reduction of Gab2-impaired UM cells migration. In the attempt to understand the biological function of Gab2 in UM, we performed cell chemotaxis assay and scratch assay. The chemotaxis assay showed similar results between parental MEL202 and SCR/MEL202 cells. Compared with control cells, the researchers found that siGab2/MEL202 cells showed dramatically reduced chemotaxis ability (Fig. 2A). These results indicate that Gab2 plays an important role in the chemotaxis of UM cells. The cell proliferation did not interfere with chemotaxis results in the present study because it only took $<3 \mathrm{~h}$ to complete the chemotaxis assay, shorter than the doubling time. Furthermore, we found that the migration of siGab2/92.1 and siGab2/MEL270 cells was also significantly impaired after reduction of Gab2 (Fig. 2B). In addition, scratch assay also showed a significant difference between the migration distance of control and Gab2 knockdown cells, and Gab2 knockdown cells took a longer time to fill the gap, further 
A
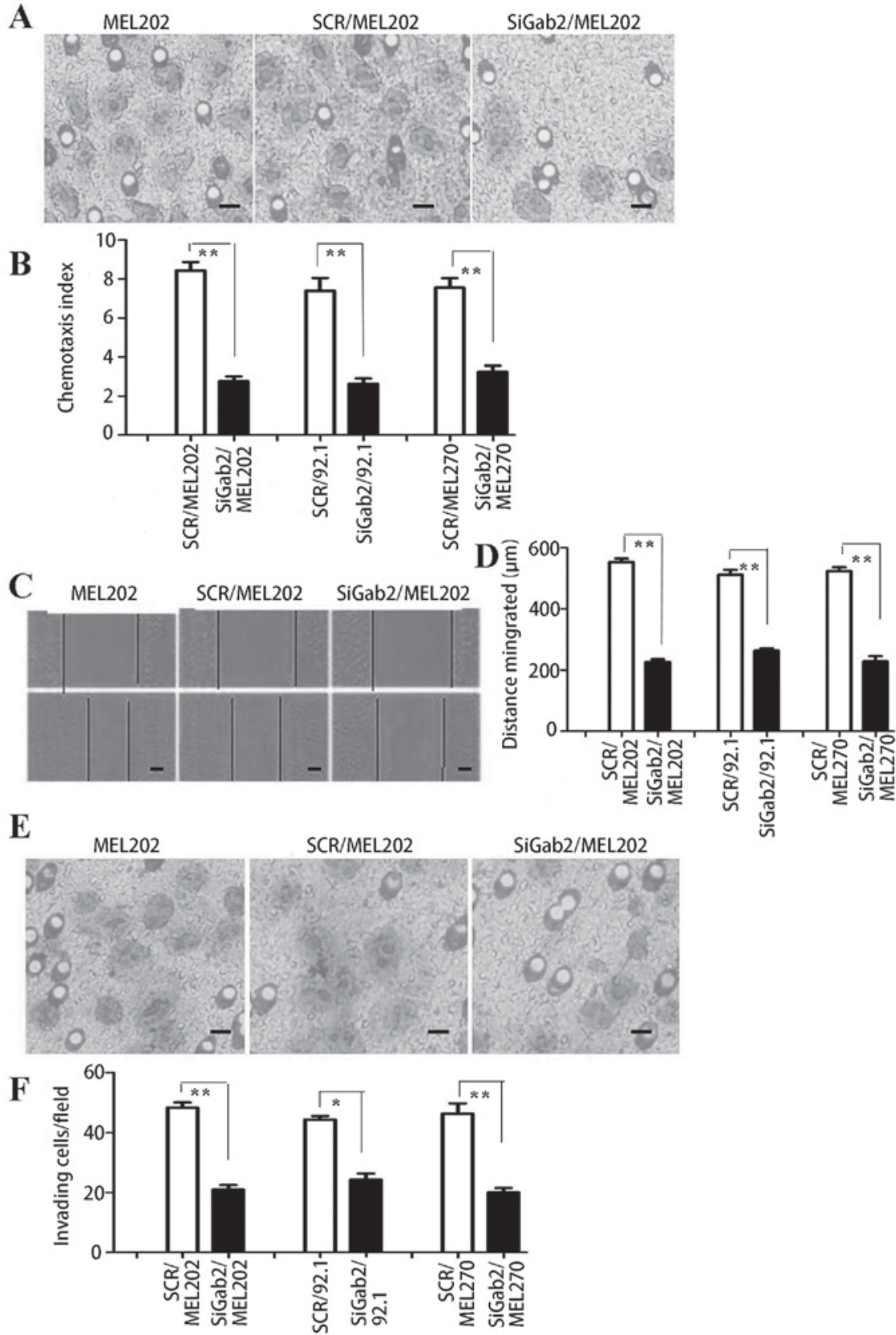

Figure 2. Downregulation of Gab2 inhibited the migration and invasion of UM cells. (A) Representative pictures of EGF-induced chemotaxis of MEL202, SCR/MEL202, and SiGab2/MEL202 cells (scale bar, $50 \mu \mathrm{m}$ ). (B) Quantification of indicated cells analyzed in chemotactic responses ("P<0.01). (C) Directional movement of MEL202, SCR/MEL202, and siGab2/MEL202 cells by scratch assay (scale bar, $20 \mu \mathrm{m}$ ). (D) Comparison of the distance migrated of the indicated cells analyzed in scratch assay. Each data point was an average of triplicate assays. (E) Representative pictures of penetrated cells in Transwell invasion assay, (scale bar, $50 \mu \mathrm{m}$ ). (F) Invasiveness of indicated cells analyzed by Transwell invasion assay. ${ }^{*} \mathrm{P}<0.01$ (two-way ANOVA).

supporting that Gab2 plays an important role in directional migration of UM cells (Fig. 2C, D).

Knockdown of Gab2 impaired UM cells invasion. Tumor cell migration is essential in cell invasion; hence, we hypothesize that Gab2 has a crucial effect on UM cells invasion. Transwell matrix penetration assay was used to test the invasiveness of the Gab2 reduction and the control cells. EGF $(10 \mathrm{ng} / \mathrm{ml})$ was used as a chemoattractant to stimulate the cells to penetrate through the Matrigel and to migrate through the filters. Compared with the control cells, the researchers found that siGab2/MEL202 cells showed dramatically reduced invasive ability (Fig. 2E). In addition, the invasiveness of siGab2/MEL270, siGab2/92.1 cells was also significantly impaired after reduction of Gab2 (Fig. 2F).

Knockdown of Gab2 led to decreased expression of MMP-2 and MMP-9. We have proven that Gab2 knockdown can reduce the invasive ability of UM cells. Given that MMPs are involved in tumor invasion and metastasis (14-16), we focused on detection of MMPs expression in Gab2 reduction and control cells. ELISA assay showed that the expression levels of MMP-2 and MMP-9 were obviously decreased in Gab2 reduction cells when treated with EGF, whereas in the absence of EGF, the expression levels of MMP-2 and MMP-9 in the culture medium of Gab2 reduction cells had no obvious 
A
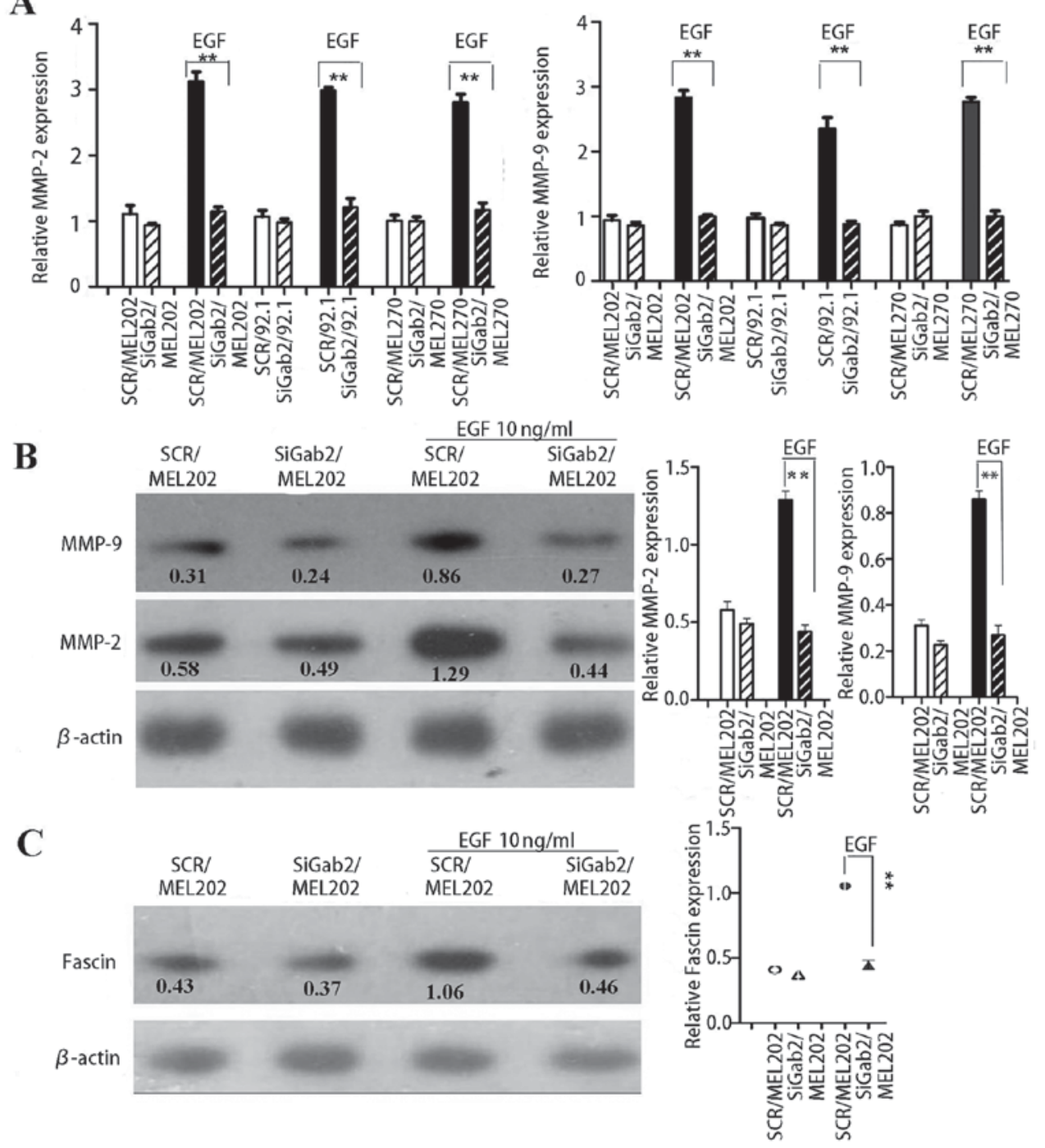

Figure 3. Knockdown of Gab2 inhibits the expression of MMP-2, MMP-9, and fascin in UM cells. (A) In the presence of EGF (10 ng/ml), the SCR/MEL202 and siGab/MEL202 cells were cultured for $48 \mathrm{~h}$ with serum-free medium on a six-well plate. After culture, the culture media were concentrated to assess MMP-2 and MMP-9 secretion using ELISA. (B) SCR/MEL202 and siGab2/MEL202 cells were treated with EGF (10 ng/ml) for 5 min. After treatment of cells, proteins were extracted and western blotting using antibody to MMP2 and MMP9 was performed. $\beta$-actin was used as a loading control. (C) SCR/MEL202 and siGab2/MEL202 cells were treated with EGF $(10 \mathrm{ng} / \mathrm{ml})$ for $5 \mathrm{~min}$. After treatment of cells, proteins were extracted and western blotting using antibody to fascin was performed. Each result is a representative from at least three independent experiments.

difference with control cells (Fig. 3A). Furthermore, western blot analysis results also showed that the siGab2/MEL202 cells expressed less MMP-2 and MMP-9 than Scr/MEL202 cells when treated with EGF (Fig. 3B). These results strongly suggested that Gab2 was closely relevant to MMP-2 and MMP-9 expression in UM cells.

Knockdown of Gab2 led to decreased expression of fascin. The actin-bundling protein fascin (FSCN1) is a marker of many malignancy tumors and is important for migration and invasion of tumor cells $(17,18)$. However, the roles of fascin in the Gab2-induced malignant behaviors of UM remain unclear. In the present study, western blot analysis results showed that fascin levels were evidently higher in SCR/MEL202 cells than that in SiGab2/MEL202 cells when stimulated by EGF (10 ng/ml), while no obvious difference was found when the cells were not treated by EGF (Fig. 3C). These results indicate that knockdown of Gab2 in UM cells decreased fascin expression in cells treated with EGF and strongly suggest that Gab2 was closely relevant to fascin expression in UM cells. On the other hand, fascin might play important roles in the Gab2-induced UM invasion.

\section{Discussion}

The important finding of the present study is that Gab2 is obviously over-expressed in UM cell lines and primary UM tissues. More importantly, high Gab2 expression could be an aggressive biological feature for UM.

In vitro research showed that downregulation of Gab2 through siRNA severely impaired the migration ability of UM cells. This result suggests that Gab2 is one of the crucial factors involved in the UM cells migration. Furthermore, Gab2 knockdown obviously impaired the invasiveness of UM cells. MMPs, especially MMP-2 and MMP-9, have been proven to play vital roles in facilitating the metastasis of UM cells. 
The involvement of MMPs in cancer progression has been reported in various cancer cell types. This study displayed that with the stimulation of EGF, the Gab2-reduced cells exhibited no observable enhanced expression of MMP-2 and MMP-9 compared with control cells. Thus, we speculate that the reduction of Gab2 will lead to a drastic decreased expression of MMP-2 and MMP-9 and then finally a decrease in the invasive ability.

Previous data have suggested that fascin is concentrated in the leading edge of cancer tissues and mediates self-seeding of cancer cells (19). Tumor cells with high expression of fascin have been found to exhibit increased membrane protrusions and migration ability, suggesting that fascin is associated with clinical aggressiveness and metastasis (20). To evaluate the involvement of Gab2 in fascin expression in UM cells, fascin protein levels were tested in Gab2 knockdown and corresponding control cells. In accordance with previous studies, western blot analysis revealed that when treated with EGF, the Gab2 knockdown cells had obviously lower fascin expression than control cells.

In conclusion, we have shown that Gab2 is overexpressed in UMs and plays an important role in UM invasion. Moreover, our findings suggest a novel role for Gab2 in modulating MMP-2, MMP-9, and fascin expression in regulating the invasion of UM tumor cells. Thus, Gab2 may be a useful prognostic marker and a novel therapeutic target for UM.

\section{Acknowledgments}

The present study was supported by the National Scientific Foundation of China (grant nos. 81672631 and 81072068), Science Foundation of Shandong Province (grant nos. ZR2015HL119 and ZR2011HL047), the Science Foundation of Gansu Province (grant no. 2014GS02292), and the Science and Technology Development Plan of Weifang (grant no. 20121230).

\section{References}

1. Abbott AM, Doepker MP, Kim Y, Perez MC, Gandle C, Thomas KL, Choi J, Shridhar R and Zager JS: Hepatic progression-free and overall survival after regional therapy to the liver for metastatic melanoma. Am J Clin Oncol, 2017.

2. Augsburger JJ, Corrêa ZM and Shaikh AH: Quality of evidence about effectiveness of treatments for metastatic uveal melanoma. Trans Am Ophthalmol Soc 106: 128-37, 2008.

3. Ma YW, Liu YZ and Pan JX: Verteporfin induces apoptosis and eliminates cancer stem-like cells in uveal melanoma in the absence of light activation. Am J Cancer Res 6: 2816-2830, 2016.
4. Harbour JW: The genetics of uveal melanoma: An emerging framework for targeted therapy. Pigment Cell Melanoma Res 25: 171-181, 2012.

5. Ding C, Luo J, Li L, Li S, Yang L, Pan H, Liu Q, Qin H, Chen C and Feng J: Gab2 facilitates epithelial-to-mesenchymal transition via the MEK/ERK/MMP signaling in colorectal cancer. J Exp Clin Cancer Res 35: 5, 2016.

6. Wang Y, Sheng Q, Spillman MA, Behbakht K and Gu H: Gab2 regulates the migratory behaviors and $\mathrm{E}$-cadherin expression via activation of the PI3K pathway in ovarian cancer cells. Oncogene 31: 2512-2520, 2012.

7. Wöhrle FU, Daly RJ and Brummer T: Function regulation and pathological roles of the Gab/DOS docking proteins. Cell Commun Signal 7: 22, 2009.

8. Wang Y, Sheng Q, Spillman MA, Behbakht K and Gu H: Gab2 regulates the migratory behaviors and $\mathrm{E}$-cadherin expression via activation of the PI3K pathway in ovarian cancer cells. Oncogene 31: 2512-2520, 2012.

9. Shi L, Sun X, Zhang J, Zhao C, Li H, Liu Z, Fang C, Wang X, Zhao C, Zhang X, et al: Gab2 expression in glioma and its implications for tumor invasion. Acta Oncol 52: 1739-1750, 2013.

10. Lee SH, Jeong EG, Nam SW, Lee JY, Yoo NJ and Lee SH: Increased expression of Gab2, a scaffolding adaptor of the tyrosine kinase signalling, in gastric carcinomas. Pathology 39: 326-329, 2007.

11. Xu XL, Wang X, Chen ZL, Jin M, Yang W, Zhao GF and Li JW: Overexpression of Grb2-associated binder 2 in human lung cancer. Int J Biol Sci 7: 496-504, 2011.

12. Zhang B, Gu F, She C, Guo H, Li W, Niu R, Fu L, Zhang N and Ma Y: Reduction of Akt2 inhibits migration and invasion of glioma cells. Int J Cancer 125: 585-595, 2009.

13. Takino T, Nakada M, Miyamori H, Yamashita J, Yamada KM and Sato H: CrkI adapter protein modulates cell migration and invasion in glioblastoma. Cancer Res 63: 2335-2337, 2003.

14. Xu Z, Liu D, Fan C, Luan L, Zhang X and Wang E: DIXDC1 increases the invasion and migration ability of non-small-cell lung cancer cells via the PI3K-AKT/AP-1 pathway. Mol Carcinog 53: 917-925, 2014.

15. Weng Y, Cai M, Zhu J, Geng J, Zhu K, Jin X and Ding W: Matrix metalloproteinase activity in early-stage lung cancer. Onkologie 36: 256-259, 2013.

16. Takemoto N, Tada M, Hida Y, Asano T, Cheng S, Kuramae T, Hamada J, Miyamoto M, Kondo S and Moriuchi T: Low expression of reversion-inducing cysteine-rich protein with Kazal motifs (RECK) indicates a shorter survival after resection in patients with adenocarcinoma of the lung. Lung Cancer 58: 376-383, 2007.

17. Machesky LM and Li A: Fascin: Invasive filopodia promoting metastasis. Commun Integr Biol 3: 263-270, 2010.

18. Papaspyrou K, Brochhausen C, Schmidtmann I, Fruth K, Gouveris H, Kirckpatrick J, Mann W and Brieger J: Fascin upregulation in primary head and neck squamous cell carcinoma is associated with lymphatic metastasis. Oncol Lett 7: 2041-2046, 2014.

19. Hashimoto Y, Skacel M and Adams JC: Roles of fascin in human carcinoma motility and signaling: Prospects for a novel biomarker? Int J Biochem Cell Biol 37: 1787-1804, 2005.

20. Jayo A and Parsons M: Fascin: A key regulator of cytoskeletal dynamics. Int J Biochem Cell Biol 42: 1614-1617, 2010. 